\title{
Estimation of healthcare-related charges in women with BRCA mutations and breast cancer
}

Joseph Biskupiak ${ }^{1 *}$, Sudhir Unni ${ }^{2}$, Claire Telford ${ }^{3}$, Minkyoung Yoo ${ }^{1}$, Xiangyang Ye ${ }^{1}$, Rishi Deka ${ }^{4}$, Diana Brixner ${ }^{1}$ and David Stenehjem ${ }^{1,5}$

\begin{abstract}
Background: Breast cancer costs were estimated at $\$ 16.5$ billion in 2010 and were higher than other cancer costs. There are limited studies on breast cancer charges and costs by BRCA mutations and receptor status. We examined overall health care and breast cancer-related charges by BRCA status (BRCAm vs. BRCAwt), receptor status (HER2+ vs. HER2-), and treatment setting (neoadjuvant vs. adjuvant).

Methods: Retrospective cohort study of charge data from 1995-2014 in an academic medical center. Facilities, physician, pharmacy, and diagnosis-related charges were presented as mean and median charges with standard deviation (SD) and interquartile ranges (25\%-75\%). Wilcoxon rank-sum test was used to assess statistically significant differences in charges between comparators.

Results: Total median breast-cancer related charges were $\$ 65,414$ for BRCAm and $\$ 54,635$ for BRCAwt ( $p=0.19$ ); however all-cause charges were higher for BRCAm patients $(\$ 145,066$ vs. $\$ 119,119, p<0.001)$. HER2+ status was associated with higher median breast cancer charges $(\$ 152,159$ vs. $\$ 44,087, p<0.0001)$ that was driven by the charges for biological agents. Patients initially seen in the neoadjuvant setting had higher mean breast cancer charges than in the adjuvant setting $(\$ 117,922$ vs. $\$ 80,061, p<0.0001)$.

Conclusion: BRCA mutation status was not associated with higher breast cancer charges but HER2+ status had significantly higher charges, due to charges for biological agents. Patients who initially received neoadjuvant treatment had significantly higher overall treatment charges than adjuvant therapy patients. With the advent of novel therapies for BRCAm, the economic impact of these treatments will be important to consider relative to their survival benefits.
\end{abstract}

Keywords: Breast cancer, BRCA, HER-2, Charges

* Correspondence: Joseph.Biskupiak@pharm.utah.edu

'Department of Pharmacotherapy, Outcomes Research Center, University of Utah, Salt Lake City, USA

Full list of author information is available at the end of the article

(c) The Author(s). 2021 Open Access This article is licensed under a Creative Commons Attribution 4.0 International License, which permits use, sharing, adaptation, distribution and reproduction in any medium or format, as long as you give appropriate credit to the original author(s) and the source, provide a link to the Creative Commons licence, and indicate if changes were made. The images or other third party material in this article are included in the article's Creative Commons licence, unless indicated otherwise in a credit line to the material. If material is not included in the article's Creative Commons licence and your intended use is not permitted by statutory regulation or exceeds the permitted use, you will need to obtain permission directly from the copyright holder. To view a copy of this licence, visit http://creativecommons.org/licenses/by/4.0/ The Creative Commons Public Domain Dedication waiver (http://creativecommons.org/publicdomain/zero/1.0/) applies to the data made available in this article, unless otherwise stated in a credit line to the data. 


\section{Background}

Breast cancer is the most common type of cancer observed in women in the US and is associated with significant clinical and economic burden [1]. In 2015, there were nearly 3.5 million women living in the US with a breast cancer diagnosis and it is estimated that approximately $12.4 \%$ of women in the US will receive a breast cancer diagnosis at some point in their lives [1].

Breast cancer is associated with significant costs. In 2010, breast cancer treatment costs in the US were approximately $\$ 16.5$ billion, which was higher than those for any other cancer and these costs are expected to rise to $\$ 20.5$ billion by 2050 [2]. Similar results have also been observed in Europe wherein breast cancer costs accounted for the highest healthcare costs of all cancers [3]. The published economic studies in breast cancer vary widely by perspective, methodology, time horizon, and patient populations [4]. While breast cancer costs have been estimated by tumor stage, time after diagnosis, and treatment options, there are limited studies on such costs categorized by BRCA mutations and receptor status as well as neoadjuvant and adjuvant treatment settings [4-6].

The assessment of costs within a healthcare system is challenging given the complex interplay between costs, charges, and reimbursed amounts for the service provided [7]. In many instances, it is difficult to access costs due to their proprietary nature, which is dependent on the contractual agreements between the healthcare systems and payer organizations. Studies have used costto-charge ratios, the ratio of charges over CMS allowable costs, to describe the costs assigned by the healthcare system. However, there are significant variations in these cost estimates depending on the type of institution and geographical location $[8,9]$. Thus, healthcare studies typically use the more readily available charge data to make economic assessments.

The purpose of this study was to explore cumulative health care charges in women diagnosed with breast cancer and tested for BRCA mutations with charges stratified by BRCA mutation status, receptor status, and treatment settings.

\section{Methods}

\section{Study design, population, and data source}

This was a retrospective cohort study utilizing charge data from 1995 to 2014 in the University of Utah Clinical Enterprise Data Warehouse (EDW), a repository containing records for more than 3.3 million patients dating back to 1995. The EDW contains data from electronic medical records, laboratory and radiology findings, administrative claims, and patient encounter data. Patients included in the study were adult women with breast cancer who were tested for BRCA mutations (BRCA positive [BRCAm] or
BRCA wildtype [BRCAwt]) and received treatment at the Huntsman Cancer Institute (HCI) in Salt Lake City, Utah. $\mathrm{HCI}$ is the major cancer center in the Intermountain West region as well as a National Cancer Institute-Designated Cancer Center and member of the National Comprehensive Cancer Network. Patients were identified from the Huntsman Cancer Institute Tumor Registry (HCI-TR) using site (ICD-O C50.x) and histology codes for the diagnosis of breast cancer between January 1, 1995 and December 31, 2014. To our knowledge, healthcare providers from $\mathrm{HCI}$ had unrestricted availability to request BRCA testing during the study period. The date of breast cancer diagnosis was defined as the index date. Electronic medical records of eligible patients were manually reviewed to identify and obtain information regarding BRCA mutation, HER-2, and ER/PR status, treatment patterns, and disease progression. Chart abstraction was conducted by an oncology clinical pharmacist using a chart abstraction form and reviewed by another oncology clinical pharmacist. Demographic and clinical characteristics of these patients were obtained through a EDW query.

Patients were excluded from the study if they were < 18 years of age at the time of breast cancer diagnosis, had $<2$ encounters (clinic visits) separated by $\geq 30$ days with relevant ICD-9 codes for breast cancer in the EDW, were male patients, were not tested for a BRCA mutation or had unknown BRCA mutation status, or had no charge data.

The charge amount was the actual amount charged by the facility based on the International Classification of Diseases, 9th Clinical Modification (ICD-9-CM) diagnosis and Current Procedural Terminology (CPT) codes. The estimated yearly charges were adjusted for inflation to 2014 values using the Bureau of Labor Statistics Consumer Price Index for Medical Care [10].

\section{Study outcomes}

The patients identified in this study were linked to the administrative data in the EDW to obtain all charge data related to the healthcare utilization from the index date until they were lost to follow-up (no additional encounters in the database or death occurred). Charges (the monetary amount billed for services provided to a patient) were initially categorized based on paid claims linked to the corresponding ICD-9 and CPT codes as breast cancer-related, other (non-breast) cancers related, non-cancer related, and all-cause charges. Each categorical charge was stratified as inpatient- and outpatientrelated charges based on site of care. Inpatient charges refer to charges incurred whn a patient has been admitted to a hospital for care. Outpatient charges refer to charges incurred when a patient is treated in an ambulatory clinic setting (not admitted to a hospital for care). All-cause charges were defined as the sum of all charges (breast cancer-related, other cancers related, and non- 
cancer related) incurred from index date to death or the end of study whichever occurred earlier.

Charges were also categorized as facilities/technical charges, physician/professional charges, and pharmacy charges. The pharmacy charges were sub-divided by the type of treatment provided (chemotherapy, hormonal therapy, biological therapy, targeted small molecule therapy, investigational drug, chemotherapy administration charges, and other medications).

Breast cancer-related charges were further stratified by BRCA mutation status, HER2 status, and initial treatment setting at cancer diagnosis. Initial treatment setting was defined as the first treatment for the breast cancer patient at diagnosis and included non-metastatic adjuvant therapy, non-metastatic neoadjuvant therapy, surgical resection, and treatment for metastatic disease. BRCA mutation status was categorized as BCRA mutated gene (BRCAm) and BCRA wild-type gene BCRAwt. HER2 status was categorized as HER2+ (HER-2 amplified tumors) that included ER+/PR+/HER2 + and ER-/PR-/HER2 + patients; and HER2- (HER2 non-amplified tumors) that included ER-/PR-/HER2- (also called triple negative breast cancer $[\mathrm{TNBC}])$ and $\mathrm{ER}+/ \mathrm{PR}+/ \mathrm{HER} 2-$ patients. If a patient progressed to metastatic disease after receiving adjuvant, neoadjuvant or surgical resection, their charges were assigned to the initial treatment setting until progression to metastatic disease at which point subsequent charges were assigned to the metastatic group. Hence, metastatic patients could contribute charges to more than one treatment setting; however, their charges were not double counted.

\section{Statistical analysis}

Charges were presented as mean and median charges with standard deviation (SD) and interquartile ranges (25\%-75\%). Wilcoxon rank-sum test was used to assess statistically significant difference in health care charges across comparator groups. All analyses were conducted using SAS version 9.3 (SAS Institute, Cary, NC).

\section{Results}

A total of 5,712 women had a diagnosis of breast cancer during 1995-2014 of which 835 (14.6\%) were tested for a BRCA mutation with documented results from BRCA testing (Myriad Laboratories, Inc.) The number of women undergoing BRCA testing increased from 2000 to 2014 . When categorized by 5 -year increments by year of diagnosis, $5.7 \%$ women were tested during 2000 2004, $17.1 \%$ were tested during 2005-2009, and $23.1 \%$ were tested during 2010-2014. Of the 835 patients with BRCA testing, 816 had valid charge data and were included in the study. There were 134 (16.4\%) patients with a BRCAm mutation and $682(83.6 \%)$ patients were BRCAwt. Within these groups, HER2 + tumors were observed in 15 (11.2\%) and 127 (18.6\%) patients;
HER2- tumors were seen in 91 (67.9\%) and $430(63.0 \%)$ patients; and 28 (20.9\%) BRCAm and 125 (18.3\%) BRCAwt patients had other/unknown status (Fig. 1a).

With respect to initial treatment setting after breast cancer diagnosis, $553(67.8 \%)$ patients received adjuvant therapy, 148 (18.1\%) patients received neoadjuvant therapy, and $73(8.9 \%)$ patients had surgical resection alone. There were $42(5.1 \%)$ patients who presented with metastatic breast cancer at the time of diagnosis. Of the patients who received adjuvant, neoadjuvant and surgical resection at diagnosis, 58 (10.5\%), 25 (16.9\%), and 15 (20.5\%) progressed to metastatic disease during the study period respectively. The median time to progression for these 98 patients was 3.5 years ( \pm IQR $2.2-9.7$ years). Thus, of the total 140 patients who had metastatic disease during the study period, 133 (16.3\% of 816 patients) had valid charges (Fig. 1b).

Table 1 lists the demographic and clinical characteristics of these patients. Age at diagnosis was similar between BRCAm and BRCAwt (45.9 years vs. 47.1 years) patients; breast cancer stage at diagnosis was also similar between the two groups. BRCAm patients had significantly longer median follow-up times (62.4 [ \pm 74.5] months vs. $46.6[ \pm 57.7]$ months, $p=0.0045)$, The BRCAm group had more triple negative breast cancer patients vs. BRCAwt group (28.4\% vs. $11.9 \%)$. More BRCAm patients had grade 3 histological tumor grades and conversely, more BRCAwt patients had grade 2 histology (Grade 1: $52.2 \%$ vs. $32.0 \%$; Grade $2: 38.1 \%$ vs. $45.0 \%$ ).

\section{Comparison of health care cumulative charges between BRCAm and BRCAwt patients}

Total mean (SD) breast cancer related charges were similar between BRCAm vs. BRCAwt patients, $\$ 86,689(\$ 75,937)$ vs. $\$ 85,843 \$ 97,304, p=0.19$, respectively (Table 2 ) while all-cause charges were significantly higher for BRCAm patients $(\$ 145,066[\$ 117,462]$ vs. $\$ 119,119[\$ 122,169], p<$ 0.001; Fig. 2). Facility/technical charges accounted for $40.5 \%$ and $32.5 \%$ of the total breast cancer related charges for BRCAm and BRCAwt groups (Table 2). Pharmacy charges accounted for $40.8 \%$ and $49.4 \%$ of total breast cancer charges for BRCAm and BRCAwt groups (Table 2). Chemotherapy was received by $58 \%$ of patients and accounted for $23.9 \%$ of pharmacy charges for BRCAm patients; biologics were received by $16 \%$ of patients and accounted for $43.0 \%$ of pharmacy charges for BRCAwt patients. Physician/professional charges accounted for $18.7 \%$ and $18.1 \%$ of the total breast cancer related charges for BRCAm and BRCAwt groups respectively (Table 2).

\section{Comparison of health care cumulative charges between HER2 + and HER2- patients}

Breast cancer related charges were significantly higher for HER2 + patients vs. HER2- ( $\$ 155,858$ vs. $\$ 69,883 ; p<0.001$, 


\section{a. BY BRCA and HER2 Receptor Status}

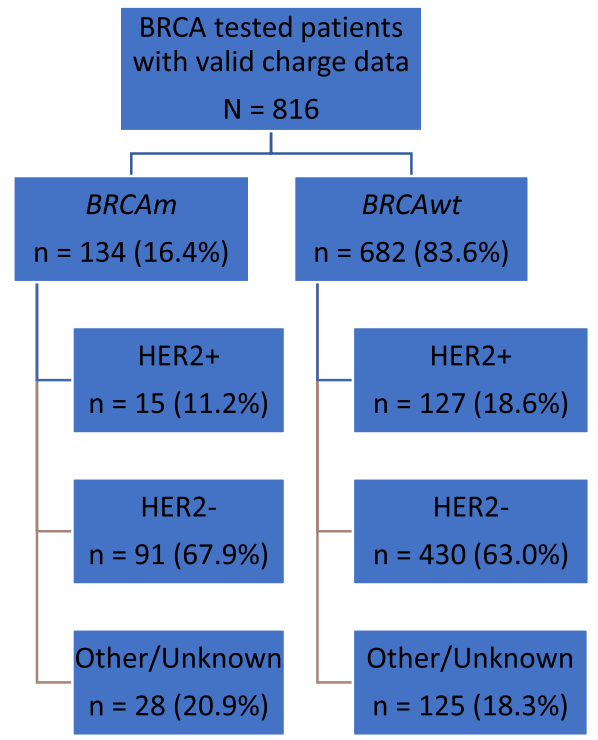

b. By Initial Treatment at Diagnosis

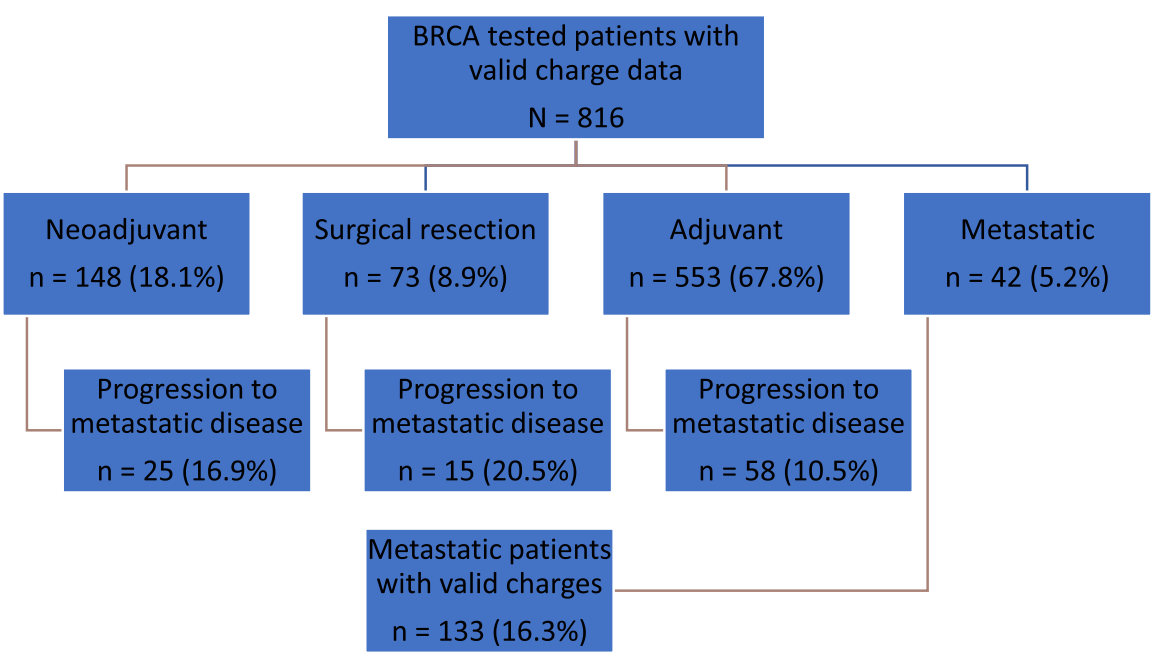

Fig. 1 Patient Identification Flow Chart . a. By BRCA and HER2 Receptor Status. b. By Initial Treatment at Diagnosis

Fig. 3) which was primarily driven by outpatient charges ( $\$ 139,322$ vs. $\$ 52,841, p<0.001$, Fig. 3). In addition, HER $2+$ patients incurred significantly higher charges across nearly all breast cancer related charge categories (Table 3), especially biologic therapy $(\$ 71,855[\$ 72,075]$ vs. $\$ 732[\$ 12$, 692], $p<0.0001$; Table 3).

\section{Comparison of health care cumulative charges by initial treatment setting}

Patients seen initially in the neoadjuvant setting had higher mean breast cancer related charges vs. those seen in the adjuvant treatment setting $(\$ 117,922$ vs. $\$ 80,061$, $p<0.0001$; Fig. 4). Patients receiving neoadjuvant treatment had significantly higher charges across most of the charge categories vs. patients receiving adjuvant treatment (Table 4). Patients with metastatic disease had a mean breast cancer related total charge of $\$ 101,969$ (Fig. 4).

\section{Discussion}

The purpose of this study was to identify the important drivers of charges in women with breast cancer who were tested for BRCA mutation status and received treatment at a National Comprehensive Cancer Network/National Cancer Institute (NCCN/NCI)-accredited 
Table 1 Demographic and Clinical Characteristics of the Study Sample

\begin{tabular}{|c|c|c|c|}
\hline Variables & BRCAm $(n=134)$ & BRCAwt $(n=682)$ & $\begin{array}{l}\text { BRCAm vs. BRCAwt } \\
p \text {-value }\end{array}$ \\
\hline \multicolumn{4}{|c|}{ Demographic Characteristics } \\
\hline Age, Mean \pm SD & $45.9 \pm 9.4$ & $47.1 \pm 11.7$ & $0.2575^{\mathrm{a}}$ \\
\hline Age, Median \pm IQR & $45.0 \pm 12.9$ & $45.9 \pm 14.7$ & $0.3853^{\mathrm{b}}$ \\
\hline \multicolumn{4}{|l|}{ Ethnicity } \\
\hline Caucasian/ White & $116(86.6 \%)$ & $598(87.5 \%)$ & $0.9377^{c}$ \\
\hline Non-White & $10(7.5 \%)$ & $47(6.9 \%)$ & \\
\hline Unknown & $8(6.0 \%)$ & $37(5.4 \%)$ & \\
\hline \multicolumn{4}{|l|}{ Plan Type } \\
\hline Commercial & $93(69.4 \%)$ & $426(62.5 \%)$ & $0.1324^{c}$ \\
\hline Medicare & $6(4.5 \%)$ & $60(8.8 \%)$ & \\
\hline Medicaid & $9(6.7 \%)$ & $31(4.6 \%)$ & \\
\hline Other/Unknown & $26(19.4 \%)$ & $165(24.2 \%)$ & \\
\hline \multicolumn{4}{|l|}{ Clinical Characteristics } \\
\hline \multicolumn{4}{|l|}{ Stage at diagnosis } \\
\hline I & $51(38.1 \%)$ & $262(38.4 \%)$ & $0.7387^{\mathrm{a}}$ \\
\hline$\|$ & $51(38.1 \%)$ & $250(36.7 \%)$ & \\
\hline III & $16(11.9 \%)$ & $107(15.7 \%)$ & \\
\hline IV & $9(6.7 \%)$ & $34(5.0 \%)$ & \\
\hline Unknown & $7(5.2 \%)$ & $29(4.3 \%)$ & \\
\hline \multicolumn{4}{|l|}{ Reeptor status } \\
\hline $\mathrm{ER}+/ \mathrm{PR}+/ \mathrm{HER} 2-$ & $53(39.6 \%)$ & $349(51.2 \%)$ & $<0.0001^{a}$ \\
\hline $\mathrm{ER}+/ \mathrm{PR}+/ \mathrm{HER} 2+$ & $7(5.2 \%)$ & $87(12.8 \%)$ & \\
\hline TNBC & $38(28.4 \%)$ & $81(11.9 \%)$ & \\
\hline ER-/PR-/HER2+ & $8(6.0 \%)$ & $40(5.9 \%)$ & \\
\hline Other/Unknown & $28(20.9 \%)$ & $125(18.3 \%)$ & \\
\hline \multicolumn{4}{|c|}{ Tumor histologic grade } \\
\hline 1 & $7(5.2 \%)$ & $115(16.9 \%)$ & $<0.0001^{\mathrm{a}}$ \\
\hline 2 & $51(38.1 \%)$ & $307(45.0 \%)$ & \\
\hline 3 & $70(52.2 \%)$ & $218(32.0 \%)$ & \\
\hline Unknown & $6(4.5 \%)$ & $42(6.2 \%)$ & \\
\hline
\end{tabular}

SD Standard Deviation, IQR Interquartile Range, TNBC Triple Negative Breast Cancer (ER-/PR-/HER2-)

${ }^{\mathrm{a}} \mathrm{T}$-Test

bWilcoxon Rank Sum Test

${ }^{\mathrm{C} C h i-S q u a r e d ~ T e s t}$

comprehensive cancer treatment center. In addition to BRCA status, the study also categorized patients by the HER2 status and type of treatment received at the time of breast cancer diagnosis. The study outcomes included comparison of breast cancer related charges between BRCAm and BRCAwt patients; HER2 + and HER2- patients; patients receiving adjuvant and neoadjuvant treatments overall, and by charge type. The study also compared the breast cancer related charges vs. charges associated with other cancer types and non-cancer related charges in these patients.
Our study suggests that BRCAm patients had significantly higher all-cause charges vs. BRCAwt patients. While overall breast cancer related charges were similar between these patients, other cancer related charges were significantly different (mean [SD] \$14,950 [\$40, $801]$ vs. $\$ 6,524$ [\$25,395], $p<0.0001)$. This difference in charges could potentially be attributed to risk-reducing surgical treatments or the development of other nonbreast cancers including ovarian cancer and fallopian tube cancer. Also, BRCAm patients had nearly 16 months longer duration of follow-up vs. BRCAwt patients. 
Table 2 Breast Cancer Related Cumulative Charges by BRCA Status and Charge Type

\begin{tabular}{|c|c|c|c|c|c|c|c|}
\hline \multirow[t]{2}{*}{ Health care charges (\$US) } & \multicolumn{2}{|l|}{ Overall $n=816$} & \multicolumn{2}{|l|}{ BRCAm $n=134$} & \multicolumn{2}{|c|}{ BRCAwt $n=682$} & \multirow{2}{*}{$\begin{array}{l}\text { BRCAm vs. } \\
\text { BRCAwt } \\
p \text {-value }\end{array}$} \\
\hline & $\begin{array}{l}\text { Mean } \\
\text { (SD) }\end{array}$ & Median (IQR) & $\begin{array}{l}\text { Mean } \\
\text { (SD) }\end{array}$ & Median (IQR) & $\begin{array}{l}\text { Mean } \\
\text { (SD) }\end{array}$ & Median (IQR) & \\
\hline Facilities/ Technical & $\begin{array}{l}29,090 \\
(26,802) 33.8 \%^{\mathrm{a}}\end{array}$ & $\begin{array}{l}24,027 \\
(34,043)\end{array}$ & $\begin{array}{l}35,130 \\
(28,878) 40.5 \%\end{array}$ & $\begin{array}{l}33,037 \\
(42,145)\end{array}$ & $\begin{array}{l}27,903 \\
(26,234) \\
32.5 \%\end{array}$ & $\begin{array}{l}22,458 \\
(32,529)\end{array}$ & 0.01 \\
\hline Physician/ Professional & $\begin{array}{l}15,615 \\
(13,126), 18.2 \%\end{array}$ & $\begin{array}{l}12,441 \\
(17,669)\end{array}$ & $\begin{array}{l}16,207 \\
(12,198), 18.7 \%\end{array}$ & $\begin{array}{l}15,624 \\
(18,967)\end{array}$ & $\begin{array}{l}15,498 \\
(13,306) \\
18.1 \%\end{array}$ & $\begin{array}{l}11,908 \\
(16,906)\end{array}$ & 0.25 \\
\hline Pharmacy & $\begin{array}{l}41,277 \\
(69,308) \\
48.0 \%\end{array}$ & $\begin{array}{l}9,313 \\
(58,716)\end{array}$ & $\begin{array}{l}35,352 \\
(51,919) 40.8 \%\end{array}$ & $\begin{array}{l}11,379 \\
(54,399)\end{array}$ & $\begin{array}{l}42,442 \\
(72,209) \\
49.4 \%\end{array}$ & $\begin{array}{l}8,930 \\
(59,659)\end{array}$ & 0.65 \\
\hline $\begin{array}{l}\text { Total Anticancer } \\
\text { Treatment }\end{array}$ & $\begin{array}{l}24,616 \\
(56,779)\end{array}$ & $\begin{array}{l}2,018 \\
(15,804)\end{array}$ & $\begin{array}{l}16,821 \\
(38,088)\end{array}$ & $\begin{array}{l}2,756 \\
(14,673)\end{array}$ & $\begin{array}{l}26,148 \\
(59,671)\end{array}$ & $\begin{array}{l}1,824 \\
(15,995)\end{array}$ & 0.72 \\
\hline Other Medication(s) & $\begin{array}{l}16,661 \\
(24,471)\end{array}$ & $\begin{array}{l}2,911 \\
(27,834)\end{array}$ & $\begin{array}{l}18,531 \\
(24,484)\end{array}$ & $\begin{array}{l}6,035 \\
(30,729)\end{array}$ & $\begin{array}{l}16,294 \\
(24,470)\end{array}$ & $\begin{array}{l}2,609 \\
(26,434)\end{array}$ & 0.19 \\
\hline Clinical Trial & $\begin{array}{l}56 \\
(347)\end{array}$ & $\begin{array}{l}0 \\
(0)\end{array}$ & $\begin{array}{l}89 \\
(621)\end{array}$ & $\begin{array}{l}0 \\
(0)\end{array}$ & $\begin{array}{l}49 \\
(262)\end{array}$ & $\begin{array}{l}0 \\
(0)\end{array}$ & 0.93 \\
\hline Chemotherapy & $\begin{array}{l}7,171 \\
(14,088)\end{array}$ & $\begin{array}{l}1,372 \\
(6,575)\end{array}$ & $\begin{array}{l}8,439 \\
(14,791)\end{array}$ & $\begin{array}{l}2,344 \\
(9,463)\end{array}$ & $\begin{array}{l}6,922 \\
(13,944)\end{array}$ & $\begin{array}{l}1,180 \\
(6,124)\end{array}$ & 0.13 \\
\hline Hormonal Therapy & $\begin{array}{l}890 \\
(5,521)\end{array}$ & $\begin{array}{l}0 \\
(0)\end{array}$ & $\begin{array}{l}769 \\
(4,334)\end{array}$ & $\begin{array}{l}0 \\
(0)\end{array}$ & $\begin{array}{l}914 \\
(5,728)\end{array}$ & $\begin{array}{l}0 \\
(0)\end{array}$ & 0.53 \\
\hline Biologic Medicine & $\begin{array}{l}16,499 \\
(50,091)\end{array}$ & $\begin{array}{l}0 \\
(0)\end{array}$ & $\begin{array}{l}7,524 \\
(30,898)\end{array}$ & $\begin{array}{l}0 \\
(0)\end{array}$ & $\begin{array}{l}18,262 \\
(52,891)\end{array}$ & $\begin{array}{l}0 \\
(0)\end{array}$ & 0.02 \\
\hline Chemotherapy Administration & $\begin{array}{l}0 \\
(5)\end{array}$ & $\begin{array}{l}0 \\
(0)\end{array}$ & $\begin{array}{l}1 \\
(7)\end{array}$ & $\begin{array}{l}0 \\
(0)\end{array}$ & $\begin{array}{l}0 \\
(5)\end{array}$ & $\begin{array}{l}0 \\
(0)\end{array}$ & NA \\
\hline Total Charges & $\begin{array}{l}85,982 \\
(94,087)\end{array}$ & $\begin{array}{l}55,230 \\
(94,787)\end{array}$ & $\begin{array}{l}86,689 \\
(75,937)\end{array}$ & $\begin{array}{l}65,414 \\
(95,894)\end{array}$ & $\begin{array}{l}85,843 \\
(97,304)\end{array}$ & $\begin{array}{l}54,635 \\
(94,085)\end{array}$ & 0.19 \\
\hline
\end{tabular}

aPercent contribution of the charge type to total charges $S D$ Standard deviation, IQR Interquartile range

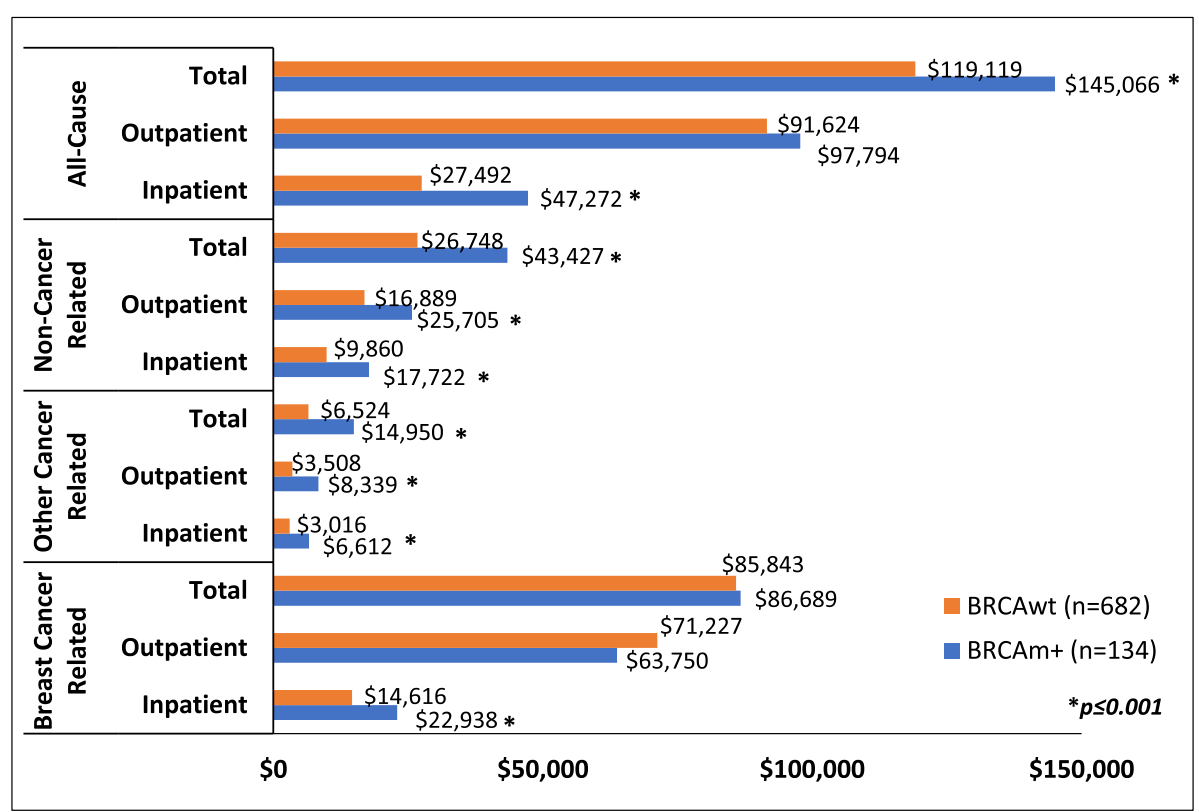

Fig. 2 Mean Cumulative Health Care Charges by BRCA Status 


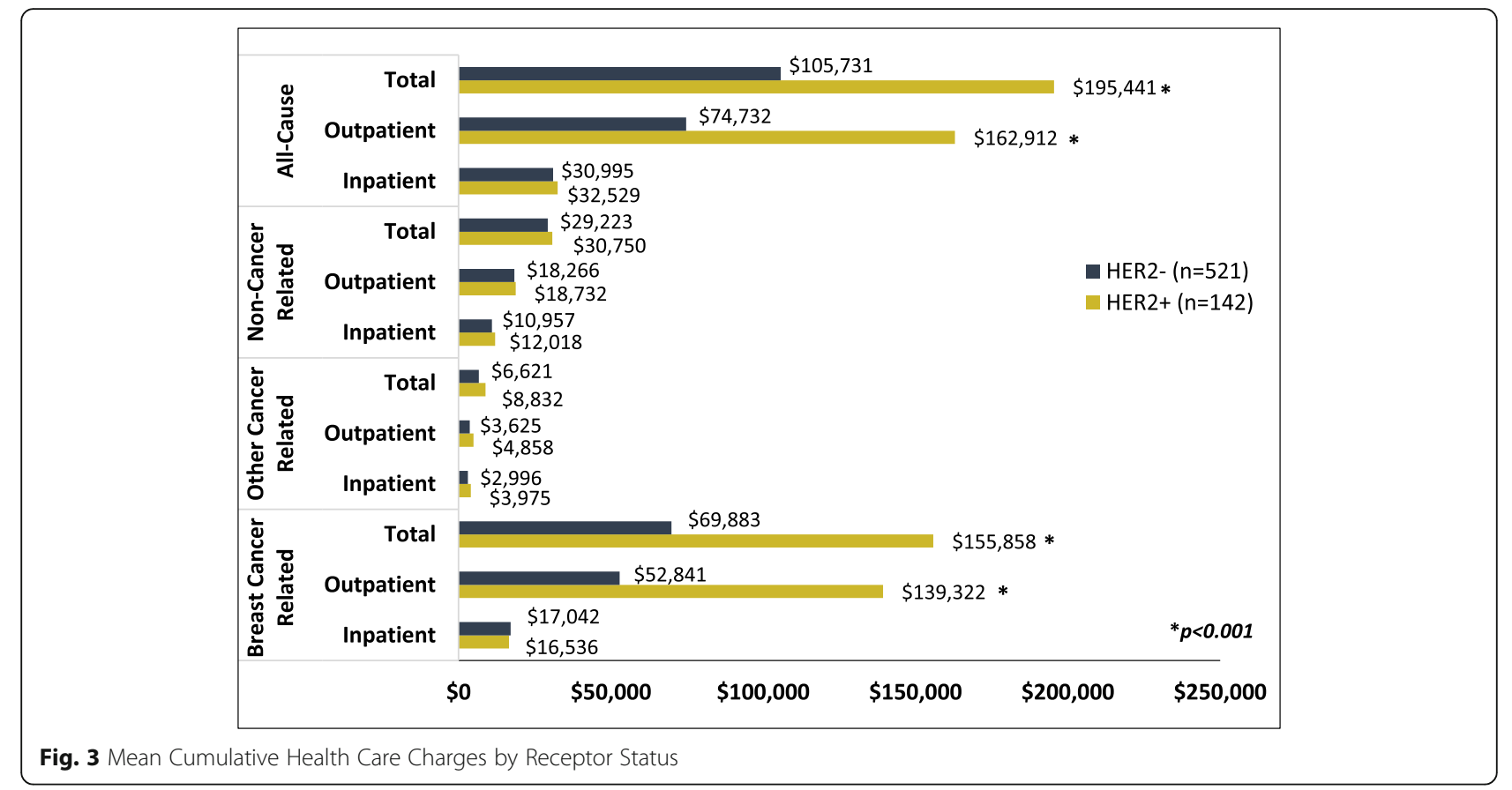

Table 3 Breast Cancer Related Cumulative Charges by Receptor Status and Charge Type

\begin{tabular}{|c|c|c|c|c|c|}
\hline \multirow[t]{2}{*}{ Health care charges (\$US) } & \multicolumn{2}{|c|}{$\begin{array}{l}\text { HER2 }+ \\
\text { ER+/PR+/HER2 + or } \\
\text { ER-/PR-/HER2 + } \\
n=142\end{array}$} & \multicolumn{2}{|c|}{$\begin{array}{l}\text { HER2- } \\
\text { TNBC or ER+/PR+/HER2- } \\
n=521\end{array}$} & \multirow{2}{*}{$\begin{array}{l}\text { HER2 + vs. HER2- } \\
\text { p-value }\end{array}$} \\
\hline & $\begin{array}{l}\text { Mean } \\
\text { (SD) }\end{array}$ & $\begin{array}{l}\text { Median } \\
\text { (IQR) }\end{array}$ & $\begin{array}{l}\text { Mean } \\
\text { (SD) }\end{array}$ & $\begin{array}{l}\text { Median } \\
\text { (IQR) }\end{array}$ & \\
\hline Facilities/ Technical & $\begin{array}{l}36,523 \\
(28,082)\end{array}$ & $\begin{array}{l}34,647 \\
(39,683)\end{array}$ & $\begin{array}{l}28,707 \\
(26,418)\end{array}$ & $\begin{array}{l}24,207 \\
(32,460)\end{array}$ & 0.00 \\
\hline Physician/ Professional & $\begin{array}{l}18,191 \\
(14,195)\end{array}$ & $\begin{array}{l}16,138 \\
(17,411)\end{array}$ & $\begin{array}{l}15,648 \\
(12,973)\end{array}$ & $\begin{array}{l}12,577 \\
(17,458)\end{array}$ & 0.04 \\
\hline Pharmacy & $\begin{array}{l}101,145 \\
(94,243)\end{array}$ & $\begin{array}{l}10,1374 \\
(147,132)\end{array}$ & $\begin{array}{l}25,529 \\
(39,646)\end{array}$ & $\begin{array}{l}7,303 \\
(42,884)\end{array}$ & $<0.0001$ \\
\hline Total Anticancer Treatment & $\begin{array}{l}82,890 \\
(81,269)\end{array}$ & $\begin{array}{l}81,783 \\
(127,300)\end{array}$ & $\begin{array}{l}7,929 \\
(21,782)\end{array}$ & $\begin{array}{l}1,364 \\
(6,006)\end{array}$ & $<0.0001$ \\
\hline Clinical Trial & $\begin{array}{l}96 \\
(430)\end{array}$ & $\begin{array}{l}0 \\
(0)\end{array}$ & $\begin{array}{l}39 \\
(199)\end{array}$ & $\begin{array}{l}0 \\
(0)\end{array}$ & 0.26 \\
\hline Chemotherapy & $\begin{array}{l}10,443 \\
(15,582)\end{array}$ & $\begin{array}{l}4,417 \\
(14,415)\end{array}$ & $\begin{array}{l}6,222 \\
(13,642)\end{array}$ & $\begin{array}{l}1,074 \\
(5,461)\end{array}$ & $<0.0001$ \\
\hline Hormonal Therapy & $\begin{array}{l}496 \\
(3,889)\end{array}$ & $\begin{array}{l}0 \\
(0)\end{array}$ & $\begin{array}{l}935 \\
(5,660)\end{array}$ & $\begin{array}{l}0 \\
(0)\end{array}$ & 0.46 \\
\hline Biologic Therapy & $\begin{array}{l}71,855 \\
(72,075)\end{array}$ & $\begin{array}{l}6,5471 \\
(117,006)\end{array}$ & $\begin{array}{l}732 \\
(12,692)\end{array}$ & $\begin{array}{l}0 \\
(0)\end{array}$ & $<0.0001$ \\
\hline Other Medication(s) & $\begin{array}{l}18,255 \\
(21,988)\end{array}$ & $\begin{array}{l}6,517 \\
(29,473)\end{array}$ & $\begin{array}{l}17,600 \\
(25,321)\end{array}$ & $\begin{array}{l}2,953 \\
(29,228)\end{array}$ & 0.02 \\
\hline
\end{tabular}

SD Standard deviation, IQR Interquartile range 


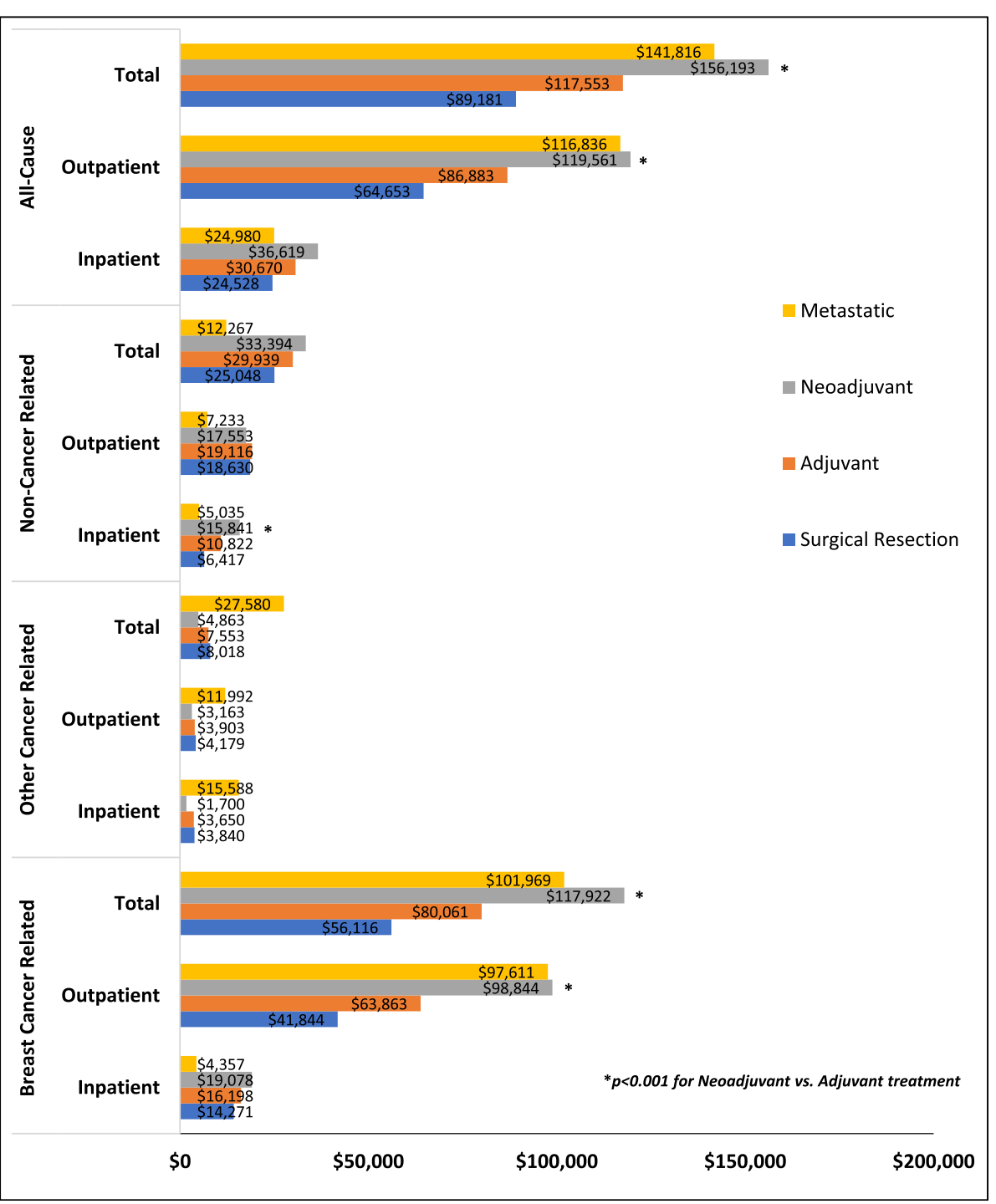

Fig. 4 Mean Cumulative Health Care Charges by Initial Treatment Setting

We are unaware of any other studies assessing economic burden of any cancer based on BRCA mutation status. Thus, clinicians, health plans, and researchers may need to consider the difference in follow-up times and identify the drivers of the costs when comparing overall costs in these patients.

HER2 + patients incurred significantly higher charges vs. HER2- patients across all charge categories which was primarily driven by biologic therapy ( $\$ 71,885$ vs. $\$ 732 ; p<0.0001)$. Patients who received neoadjuvant treatment after breast cancer diagnosis incurred the highest charges $(\$ 117,922)$, followed by those with metastatic disease $(\$ 101,969)$, and patients who received adjuvant treatment $(\$ 80,061)$.

Due to the variations in perspective, study population, year of cost basis, and duration of follow-up, for published breast cancer cost of illness studies, it is difficult to directly compare the costs for this study with existing literature. We did not observe previous studies that compared costs between BRCAm and BRCAwt or HER2 + and HER2- breast cancer patients; however, there are studies that have estimated costs by breast cancer stage and type of treatment. A recently published study by Blumen et al. estimated the overall per-patient allowed cost during the first year after breast cancer diagnosis to be $\$ 85,772$ which is similar to the average mean charges observed in this study for BRCAm and BRCAwt (\$85,982).[11] The Blumen study estimated costs by cancer stage and aggregated the costs across inpatient, outpatient, surgery, chemotherapy, radiation, prescription, and professional costs in a commercial claims database. The breast cancer treatment-related 
Table 4 Breast Cancer Total Cumulative Charges by Initial Treatment at Diagnosis and Charge Type

\begin{tabular}{|c|c|c|c|c|c|c|c|}
\hline \multirow[t]{2}{*}{ Health care charges (\$US) } & \multicolumn{2}{|c|}{ Surgical Resection $n=73$} & \multicolumn{2}{|c|}{ Adjuvant $n=553$} & \multicolumn{2}{|c|}{ Neoadjuvant $n=148$} & \multirow{2}{*}{$\begin{array}{l}\text { Adjuvant vs. } \\
\text { Neoadjuvant } \\
p \text {-value }\end{array}$} \\
\hline & Mean (SD) & Median (IQR) & Mean (SD) & Median (IQR) & Mean (SD) & Median (IQR) & \\
\hline \multicolumn{8}{|l|}{ Breast Cancer related } \\
\hline Facilities/ Technical & $\begin{array}{l}23,472 \\
(21,512)\end{array}$ & $\begin{array}{l}19,840 \\
(28,345)\end{array}$ & $\begin{array}{l}27,486 \\
(24,400)\end{array}$ & $\begin{array}{l}22,456 \\
(32,113)\end{array}$ & $\begin{array}{l}35,877 \\
(27,496)\end{array}$ & $\begin{array}{l}33,984 \\
(38,937)\end{array}$ & 0.00 \\
\hline Physician/ Professional & $\begin{array}{l}12,942 \\
(10,770)\end{array}$ & $\begin{array}{l}10,840 \\
(12,678)\end{array}$ & $\begin{array}{l}15,575 \\
(13,091)\end{array}$ & $\begin{array}{l}12,449 \\
(17,812)\end{array}$ & $\begin{array}{l}17,340 \\
(12,663)\end{array}$ & $\begin{array}{l}15,536 \\
(16,820)\end{array}$ & 0.04 \\
\hline Pharmacy & $\begin{array}{l}19,702 \\
(57,840)\end{array}$ & $\begin{array}{l}993 \\
(2,650)\end{array}$ & $\begin{array}{l}37,000 \\
(66,360)\end{array}$ & $\begin{array}{l}8,531 \\
(51,815)\end{array}$ & $\begin{array}{l}64,705 \\
(79,214)\end{array}$ & $\begin{array}{l}43,009 \\
(83,327)\end{array}$ & $<0.0001$ \\
\hline Total Anticancer Treatment & $\begin{array}{l}11,491 \\
(43,165)\end{array}$ & $\begin{array}{l}0 \\
(0)\end{array}$ & $\begin{array}{l}21,564 \\
(53,960)\end{array}$ & $\begin{array}{l}2,241 \\
(13,526)\end{array}$ & $\begin{array}{l}40,941 \\
(71,342)\end{array}$ & $\begin{array}{l}4,556 \\
(57,935)\end{array}$ & 0.00 \\
\hline Clinical Trial & $\begin{array}{l}47 \\
(196)\end{array}$ & $\begin{array}{l}0 \\
(0)\end{array}$ & $\begin{array}{l}31 \\
(215)\end{array}$ & $\begin{array}{l}0 \\
(0)\end{array}$ & $\begin{array}{l}78 \\
(297)\end{array}$ & $\begin{array}{l}0 \\
(0)\end{array}$ & 0.01 \\
\hline Chemotherapy & $\begin{array}{l}3,604 \\
(12,107)\end{array}$ & $\begin{array}{l}0 \\
(0)\end{array}$ & $\begin{array}{l}7,204 \\
(14,053)\end{array}$ & $\begin{array}{l}1,759 \\
(6,352)\end{array}$ & $\begin{array}{l}7,376 \\
(10,451)\end{array}$ & $\begin{array}{l}3,905 \\
(9,278)\end{array}$ & 0.01 \\
\hline Hormonal Therapy & $\begin{array}{l}1,022 \\
(5,843)\end{array}$ & $\begin{array}{l}0 \\
(0)\end{array}$ & $\begin{array}{l}836 \\
(5,814)\end{array}$ & $\begin{array}{l}0 \\
(0)\end{array}$ & $\begin{array}{l}399 \\
(2,711)\end{array}$ & $\begin{array}{l}0 \\
(0)\end{array}$ & 0.95 \\
\hline Biologic Medicine & $\begin{array}{l}6,818 \\
(36,636)\end{array}$ & $\begin{array}{l}0 \\
(0)\end{array}$ & $\begin{array}{l}13,492 \\
(46,279)\end{array}$ & $\begin{array}{l}0 \\
(0)\end{array}$ & $\begin{array}{l}33,088 \\
(66,386)\end{array}$ & $\begin{array}{l}0 \\
(40,885)\end{array}$ & $<0.0001$ \\
\hline Chemotherapy Administration & $\begin{array}{l}2 \\
(15)\end{array}$ & $\begin{array}{l}0 \\
(0)\end{array}$ & $\begin{array}{l}0 \\
(4)\end{array}$ & $\begin{array}{l}0 \\
(0)\end{array}$ & $\begin{array}{l}0 \\
(0)\end{array}$ & $\begin{array}{l}0 \\
(0)\end{array}$ & . \\
\hline Other Medication(s) & $\begin{array}{l}8,210 \\
(21,536)\end{array}$ & $\begin{array}{l}993 \\
(2,559)\end{array}$ & $\begin{array}{l}15,436 \\
(23,472)\end{array}$ & $\begin{array}{l}2,530 \\
(24,473)\end{array}$ & $\begin{array}{l}23,764 \\
(24,934)\end{array}$ & $\begin{array}{l}12,018 \\
(42,041)\end{array}$ & $<0.0001$ \\
\hline
\end{tabular}

SD Standard deviation, IQR Interquartile range

costs during the first year after diagnosis was $\$ 47,452$ in the Blumen study vs. $\$ 41,277$ in our study. In another study, Fu et al. estimated the per-patient medical costs during the 12 months after breast cancer diagnosis to be approximately $\$ 60,000$ (2008 dollars) [12]. Other studies have estimated lifetime costs of breast cancer that have ranged from $\$ 36,926$ (1984 values)[13] to over $\$ 100,000$ (2003 values) [14].

With respect to treatment costs by type, Campbell and Ramsey, in a synthesis of published cost studies for breast cancer, noted that patients who received adjuvant therapy had significantly higher costs $(\$ 23,000-31,000)$ vs. those who did not.[4] Though not exactly comparable with our study due to differences in methodology (BRCA tested population), we observed significantly higher charges for neoadjuvant vs. adjuvant therapy $(\$ 117,922$ vs. $\$ 80,061, p<0.0001)$.

This study has a number of limitations. First, the results may not be generalizable since the results were for a single site $(\mathrm{HCI})$ in Utah with a younger, Caucasian population. Some of the high treatment costs seen in this study may be a result of the younger age of the breast cancer cohort. Also, the community practice patterns may be different from what was observed at $\mathrm{HCI}$, which is the only NCCN/NCI designated comprehensive cancer center in the Intermountain West region. Secondly, the study results were limited by the relatively small number of BRCAm patients $(n=134)$ vs. the
BRCAwt $(n=682)$ group. Thirdly, the charges were aggregated across breast cancer disease stage, hence were not comparable to the more commonly published breast cancer cost studies that assessed costs by stage.

\section{Conclusions}

Our assessment of the treatment charges for breast cancer at a single NCCN/NCI-designated comprehensive cancer center in the Intermountain West region demonstrates that BRCA mutation status was not associated with higher breast cancer charges, but were associated with significantly higher all-cause charges. However, HER2 + status was associated with higher breast cancer charges versus HER2- tumors and this was driven by the cost of biological agents. Breast cancer patients who initially received neoadjuvant treatment after diagnosis had significantly higher breast cancer related treatment charges than those who received adjuvant therapy. These economic differences by mutation, receptor and treatment setting are important to consider when evaluating the impact of targeted therapy on overall survival benefit.

\section{Abbreviations}

BRCA: Breast cancer; BRCAm: Breast cancer mutation; BRCAwt: Breast cancer wild-type phenotype; HER2+: Human epidermal growth factor receptor 2 positive; HER2-: Human epidermal growth factor receptor 2 negative; CMS: Center for Medicare \& Medicaid Services; ER: Estrogen receptor; PR: Progesterone receptor; HCl: Huntsman Cancer Institute; NCCN: National Cancer Care Network; NCl: National Cancer Institute 


\section{Acknowledgements}

The authors would like to acknowledge Alexandre H Watanabe, PharmD for his assistance with revisions to the manuscript.

\section{Authors' contributions}

$J B, S U, D S, C T$ and DB were involved in the design of the study, interpretation of the data and manuscript draft. JB and DS were involved in the acquisition of the data. MY, XY and RD were involved in the analysis of the data, interpretation of the data and the manuscript draft. All authors have approved the submitted version and agree to be personally accountable for their own contributions and to ensure that any questions related to the accuracy or integrity of any part of the work shall be appropriately investigated, resolved and the resolution documented in the literature.

\section{Funding}

Funding for this study was provided by AstraZeneca to the University of Utah. The funding body played no role in the design of the study and collection, analysis, and interpretation of data and in writing the manuscript. CT who was an employee of AstraZeneca at the time of this study, was involved in the design of the study, interpretation of the data and writing of the manuscript.

\section{Availability of data and materials}

The datasets during and/or analyzed during the current study available from the corresponding author on reasonable request.

\section{Ethics approval and consent to participate}

This study was deemed exempt by the University of Utah Institution Review Board (IRB\# 00076204). Administrative permission to access the raw data in this study was granted by The University of Utah IRB.

\section{Consent for publication}

Not applicable.

\section{Competing interests}

$J B, S U, M Y, X Y, R D, D S$ and $D B$ were all members of the University of Utah at the time of this study and received research funding for this study. CT at the time of this study was an employee of AstraZeneca.

\section{Author details}

'Department of Pharmacotherapy, Outcomes Research Center, University of Utah, Salt Lake City, USA. Daiichi-Sanyko Inc, Baskin Ridge, New Jersey, Utah, USA. ${ }^{3}$ GSK, Gaithersburg, Maryland, USA. ${ }^{4}$ University of California San Diego, La Jolla, California, USA. ${ }^{5}$ University of Minnesota, Minneapolis, Minnesota, USA.

Received: 15 July 2020 Accepted: 21 December 2020

Published online: 13 January 2021

\section{References}

1. Surveillance E, and End Results (SEER) Program (www.seer.cancer.gov) (2013) Cancer Stat Facts: Female Breast Cancer.

2. Mariotto AB, Yabroff KR, Shao Y, Feuer EJ, Brown ML. Projections of the cost of cancer care in the United States: 2010-2020. J Natl Cancer Inst. 2011; 103(2):117-28. doi:https://doi.org/10.1093/jnci/djq495.

3. Luengo-Fernandez R, Leal J, Gray A, Sullivan R. Economic burden of cancer across the European Union: a population-based cost analysis. Lancet Oncol. 2013:14(12):1165-74. https://doi.org/10.1016/S1470-2045(13)70442-X

4. Campbell JD, Ramsey SD. The costs of treating breast cancer in the US: a synthesis of published evidence. Pharmacoeconomics. 2009;27(3):199-209. doi:https://doi.org/10.2165/00019053-200927030-00003.

5. Barron JJ, Quimbo R, Nikam PT, Amonkar MM. Assessing the economic burden of breast cancer in a US managed care population. Breast Cancer Res Treat. 2008;109(2):367-77. doi:https://doi.org/10.1007/s10549-007-9650-4.

6. Capri S, Russo A. Cost of breast cancer based on real-world data: a cancer registry study in Italy. BMC Health Serv Res. 2017;17(1):84. doi:https://doi. org/10.1186/s12913-017-2006-9.

7. Arora V, Moriates C, Shah N. The Challenge of Understanding Health Care Costs and Charges. AMA J Ethics. 2015;17(11):1046-52. doi:https://doi.org/ 10.1001/journalofethics.2015.17.11.stas1-1511.
8. Park JD, Kim E, Werner RM. Inpatient Hospital Charge Variability of U.S. Hospitals. J Gen Intern Med. 2015;30(11):1627-32. doi:https://doi.org/10. 1007/s11606-015-3352-0

9. Bai G, Anderson GF. Extreme Markup: The Fifty US Hospitals With The Highest Charge-To-Cost Ratios. Health Aff (Millwood). 2015;34(6):922-8. doi: https://doi.org/10.1377/hlthaff.2014.1414.

10. Statistics BoL Consumer Price Index. - US Medical Care and Medical Care Services.

11. Blumen H, Fitch K, Polkus V. Comparison of Treatment Costs for Breast Cancer, by Tumor Stage and Type of Service. Am Health Drug Benefits. 2016;9(1):23-32.

12. Fu AZ, Jhaveri M. Healthcare cost attributable to recently-diagnosed breast cancer in a privately-insured population in the United States. J Med Econ. 2012;15(4):688-94. doi:https://doi.org/10.3111/13696998.2012.673524.

13. Baker MS, Kessler LG, Urban N, Smucker RC. Estimating the treatment costs of breast and lung cancer. Med Care. 1991;29(1):40-9.

14. Lamerato L, Havstad S, Gandhi S, Jones D, Nathanson D. Economic burden associated with breast cancer recurrence: findings from a retrospective analysis of health system data. Cancer. 2006;106(9):1875-82. doi:https://doi. org/10.1002/cncr.21824

\section{Publisher's Note}

Springer Nature remains neutral with regard to jurisdictional claims in published maps and institutional affiliations.
Ready to submit your research? Choose BMC and benefit from:

- fast, convenient online submission

- thorough peer review by experienced researchers in your field

- rapid publication on acceptance

- support for research data, including large and complex data types

- gold Open Access which fosters wider collaboration and increased citations

- maximum visibility for your research: over $100 \mathrm{M}$ website views per year

At BMC, research is always in progress.

Learn more biomedcentral.com/submissions 